\title{
Sinonasal Carcinomas with Neuroendocrine Features: Histopathological Differentiation and Treatment Outcomes
}

\author{
Liuba Soldatova $^{1}$ Raewyn G. Campbell ${ }^{1}$ Ricardo L. Carrau ${ }^{1,2}$ Daniel M. Prevedello ${ }^{1,2}$ Paul Wakely Jr. ${ }^{3}$ \\ Bradley A. Otto ${ }^{1,2}$ Leo F. Ditzel Filho ${ }^{1}$ \\ ${ }^{1}$ Department of Otolaryngology-Head and Neck Surgery, The Ohio \\ State University Wexner Medical Center, Columbus, Ohio, \\ United States \\ 2 Department of Neurological Surgery, The Ohio State University \\ Wexner Medical Center, Columbus, Ohio, United States

\begin{abstract}
Address for correspondence Ricardo L. Carrau, MD, Departments of Otolaryngology-Head and Neck Surgery and Neurological Surgery, The Ohio State University Wexner Medical Center, Starling Loving Hall, Room B221, 320 West 10th Avenue, Columbus, OH 43210, United States (e-mail: Ricardo.Carrau@osumc.edu).
\end{abstract}

${ }^{3}$ Department of Pathology, The Ohio State University Wexner Medical Center, Columbus, Ohio, United States

J Neurol Surg B 2016;77:456-465.

\author{
Abstract \\ Keywords \\ - olfactory \\ neuroblastoma \\ - esthesioneuro- \\ blastoma \\ - sinonasal \\ undifferentiated \\ carcinoma \\ - sinonasal \\ neuroendocrine \\ carcinoma \\ - sinonasal small cell \\ cancer \\ - sinonasal carcinoid \\ - sinonasal large cell \\ cancer \\ - sinonasal cancers with \\ neuroendocrine \\ features \\ - sinonasal cancers of \\ neuroectodermal \\ origin
}

Objectives Sinonasal cancers with neuroendocrine features share similar clinical, radiological, and histopathological features; however, these tumors often exhibit varying degrees of aggressive behavior presenting significant treatment challenges. The objective of this study was to report our experience with these rare malignancies and to present a review of current literature.

Methods Following institutional review board approval, the records of all patients with biopsy-proven sinonasal malignancies over a 5-year period were reviewed.

Results The study included 14 patients with olfactory neuroblastomas (ONBs), 7 patients with sinonasal undifferentiated carcinomas (SNUC), and 2 patients with sinonasal neuroendocrine carcinomas (SNEC). Histopathologic markers aided in final diagnosis, but showed variable specificity. In patients with sufficient follow-up, the 2year disease-free survival rate was $81 \%$ (9/11) for ONB and 75\% (3/4) for SNUC. Three patients developed a regional or distant recurrence (two with ONBs and one with SNUC). Two patients, one with SNEC and one with ONB, succumbed to brain radionecrosis related to proton radiation therapy.

Conclusions Overlapping clinical and histopathological features in poorly differentiated sinonasal cancers with neuroendocrine features continue to present a diagnostic challenge. Individualized assessment and treatment strategies can improve the accuracy of the initial assessment and the treatment outcomes. received

January 29, 2016

accepted

February 26, 2016

published online

April 26, 2016 (c) 2016 Georg Thieme Verlag KG

Stuttgart · New York
DOI http://dx.doi.org/ 10.1055/s-0036-1582432. ISSN 2193-6331. 


\section{Introduction}

Sinonasal malignancies with neuroendocrine features are rare head and neck tumors that can be divided into three major histological phenotypes: olfactory neuroblastoma (ONB), sinonasal undifferentiated carcinoma (SNUC), and sinonasal neuroendocrine carcinoma (SNEC). SNEC meets all of the diagnostic criteria that define neuroendocrine tumors, while ONB and SNUC exhibit some neuroendocrine features.

The defining features for neuroendocrine neoplasms continue to be a source of ongoing debate due to the fact that some neuroendocrine markers are nonspecific and can be expressed in nonneuroendocrine malignancies. Bell et al reported the following features as diagnostic for neuroendocrine malignancies:

- Histological features of neuroendocrine differentiation (stippled chromatin, rosette formation, organoid, and trabecular growth)

- Focal or widespread expression of immunohistochemical neuroendocrine markers with synaptophysin and chromogranin being more specific

- ome degree of epithelial differentiation as evidenced by the expression of cytokeratin ${ }^{1}$

SNEC is the only true "neuroendocrine" tumor as it meets all of the criteria required for diagnosis of neuroendocrine malignancies. ONBs are considered to be neuroectodermal in origin and are classified as such in the World Health Organization (WHO) classification of head and neck tumors ${ }^{2}$; however, these tumors share some of the features present in neuroendocrine neoplasms and are often difficult to distinguish from SNECs. ${ }^{3}$ SNUCs can present with weak focal positivity for neuroendocrine markers, but in most cases completely lack any evidence of neuroendocrine markers. ${ }^{4-6}$ SNUCs are considered here due to overlapping morphology with poorly differentiated ONBs and SNECs.

SNEC is an umbrella term that encompasses both typical and atypical carcinoids, as well as small cell (SmCC) and large cell neuroendocrine carcinomas. The most commonly used WHO classification published in 2005 does not recognize large cell-type SNEC ${ }^{2}$; however, the existence of these malignancies has been established in recent reviews suggesting future revisions of the existing WHO classification. ${ }^{3}$

Sinonasal malignancies with neuroendocrine features can be classified based on the cells of origin ( - Fig. 1). ONBs arise from neuroectodermal cells of the olfactory epithelium. Although, the cells of origin for SNUCs have not been identified unquestionably, SNECs and SNUCs are thought to arise from the respiratory epithelium of the sinonasal cavity. ${ }^{7,8}$

SNECs can be further subdivided based on the cell size and differentiation. ${ }^{1,8}$ Well-differentiated SNECs are also known as carcinoid tumors, while moderately differentiated SNECS are classified as atypical carcinoid tumors ( - Fig. 1). SmCC and large cell neuroendocrine carcinomas are poorly differentiated tumors of epithelial origin.

Sinonasal carcinomas with neuroendocrine features share similar clinical and radiological attributes, and their histopathology often shows poorly differentiated morphology with several overlapping features, which creates diagnostic challenges (-Fig. 2). A correct diagnosis is imperative for appropriate treatment and prognosis as these malignancies exhibit varying degrees of aggressive behavior. This study presents a case series of the sinonasal malignancies with neuroendocrine features and a review of the current literature with an emphasis on diagnosis, treatment strategies, and outcomes.

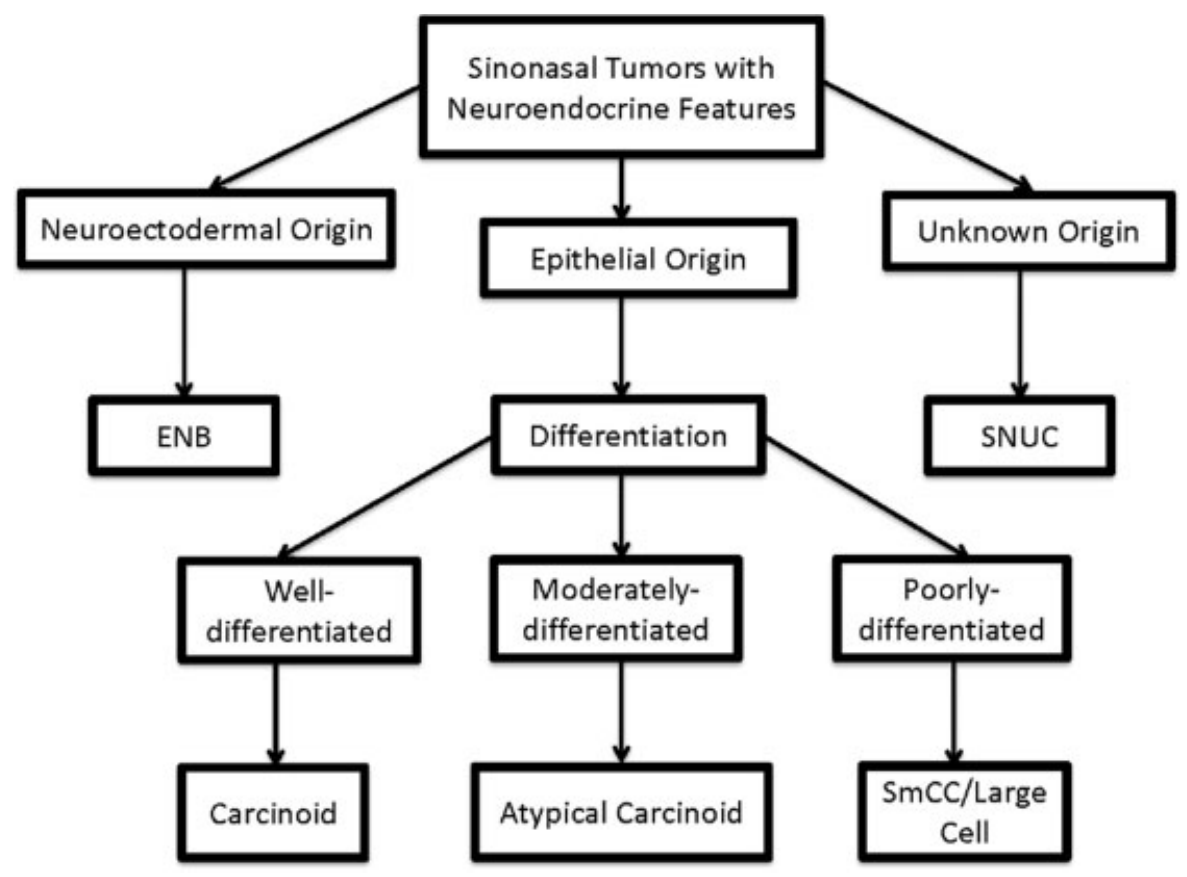

Fig. 1 Sinonasal carcinomas with neuroendocrine features: Classification. 

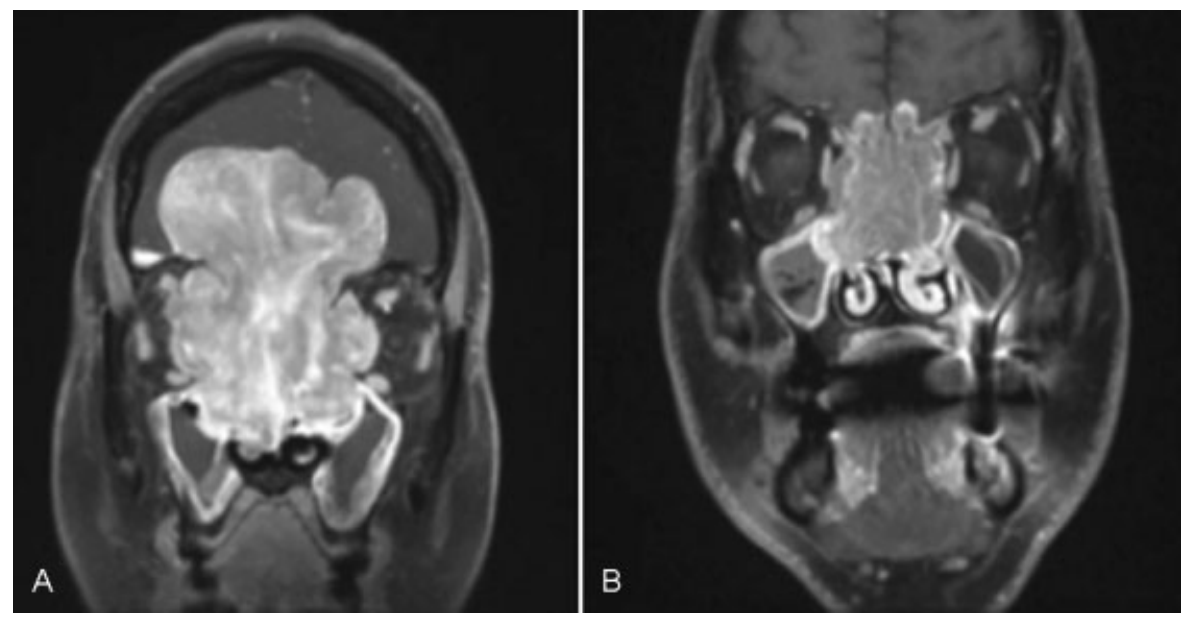

Fig. 2 Sinonasal carcinomas with neuroendocrine features. (A) T1 coronal MRI of SNUC tumor diagnosed in a 45-year-old female patient and treated with neoadjuvant chemotherapy, endoscopic surgical debulking, and proton beam therapy to the surgical bed and bilateral neck. (B) T1 coronal MRI of large cell neuroendocrine carcinoma diagnosed in a 66-year-old male patient and treated with endoscopic gross total resection followed by chemotherapy and proton beam radiation to the surgical bed, bilateral neck, and supraclavicular fossa. MRI, magnetic resonance imaging; SNUC, sinonasal undifferentiated carcinomas.

\section{Patients and Methods}

Following approval by the institutional review board, medical records of the patients treated by the skull base team at the Ohio State University James Cancer Hospital were reviewed over a 5-year period. Records of histopathologically confirmed sinonasal carcinomas with neuroendocrine features were selected. All three types of carcinomas were staged using Kadish, Dulguerov/University of California, Los Angeles (UCLA) staging systems and the 7th American Joint Committee on Cancer (AJCC) TNM staging classification of the nasal cavity and paranasal sinuses. Length of follow-up was calculated from the last treatment date to the date of last follow-up visit.

\section{Results}

We identified 14 patients with ONBs, 7 patients with SNUCs, and 2 patients with SNECs in 7 women and 16 men. Records were reviewed with emphasis on diagnosis, tumor stage, treatment, and outcomes. One ONB, one SNEC, and three SNUC specimens required expert pathological consultation at another institution due to diagnostic challenges.

ONB patients presented with Kadish stages ranging from $A$ to D (2/14 Kadish A, 4/14 Kadish B, 5/14 Kadish C, and 2/14 Kadish D), Dulguerov UCLA staging T1-T3, or AJCC 7th TNM staging T1-T4b. One ONB patient was initially treated at another institution with no information about the initial tumor stage. Nine of the 14 patients with ONB were found to have low-grade tumors (Hyams grade I-II), and 5/14 had high-grade (Hyams grade III) lesions (-Table 1).

All patients with SNUC presented at advanced stages T4aT4b by AJCC 7th TNM staging system, with assigned Dulguerov UCLA T2-T4 stages and Kadish C-D stage (5/7 Kadish C stage and 2/7 Kadish D). The SNEC cases were poorly differentiated malignancies, one T4a large cell neuroendocrine type carcinoma, and the other a T4b SmCC (-Table 2).
At the time of diagnosis, two patients with ONB and two patients with SNUC had cervical node metastases. No distant metastases were noted at the time of diagnosis in any of the patients included in this study.

In patients diagnosed before April 2013 (11 of ONBs, 4 of SNUCs), the 2-year disease-free survival rates were $81 \%(9 / 11)$ for those with ONB and 75\% (3/4) for those with SNUC. One of the patients with SNUC presented with a T4bN2cM0, an AJCC Stage IVB tumor that was treated with surgery followed by chemoradiation. This patient developed distant metastases to the brain and the bone 5.5 months after the initial treatment. These lesions were treated with radiation therapy; nonetheless, the patient passed away 26 months after treatment due to extensive distant metastases to the brain and bones. One patient with large cell type SNEC succumbed to treatment complications 21 months after the treatment due to brain radionecrosis. Of patients treated at our institution, two patients with ONB and one with SNUC suffered a regional or distant recurrence (i.e., no local recurrence was encountered).

\section{Discussion}

\section{Histopathological Diagnosis}

Sinonasal malignancies with neuroendocrine features cannot be distinguished based on clinical presentation or radiological studies. These malignancies always require histopathological assessment of the specimen by an expert pathologist. Under light microscopy, well-differentiated ONBs are composed of homogenous small-to-medium size cells with round to oval nuclei, sparse cytoplasm, punctate chromatin, small or absent nucleoli and fibrillary cytoplasmic material. .,8 $^{\text {, }}$

Initially, ONBs were described as tumors with rosette or pseudorosette cell arrangement. ${ }^{9}$ Presence of rosettes or pseudorosettes and ganglion cells is one of the considerations for the most commonly used ONB grading system introduced 


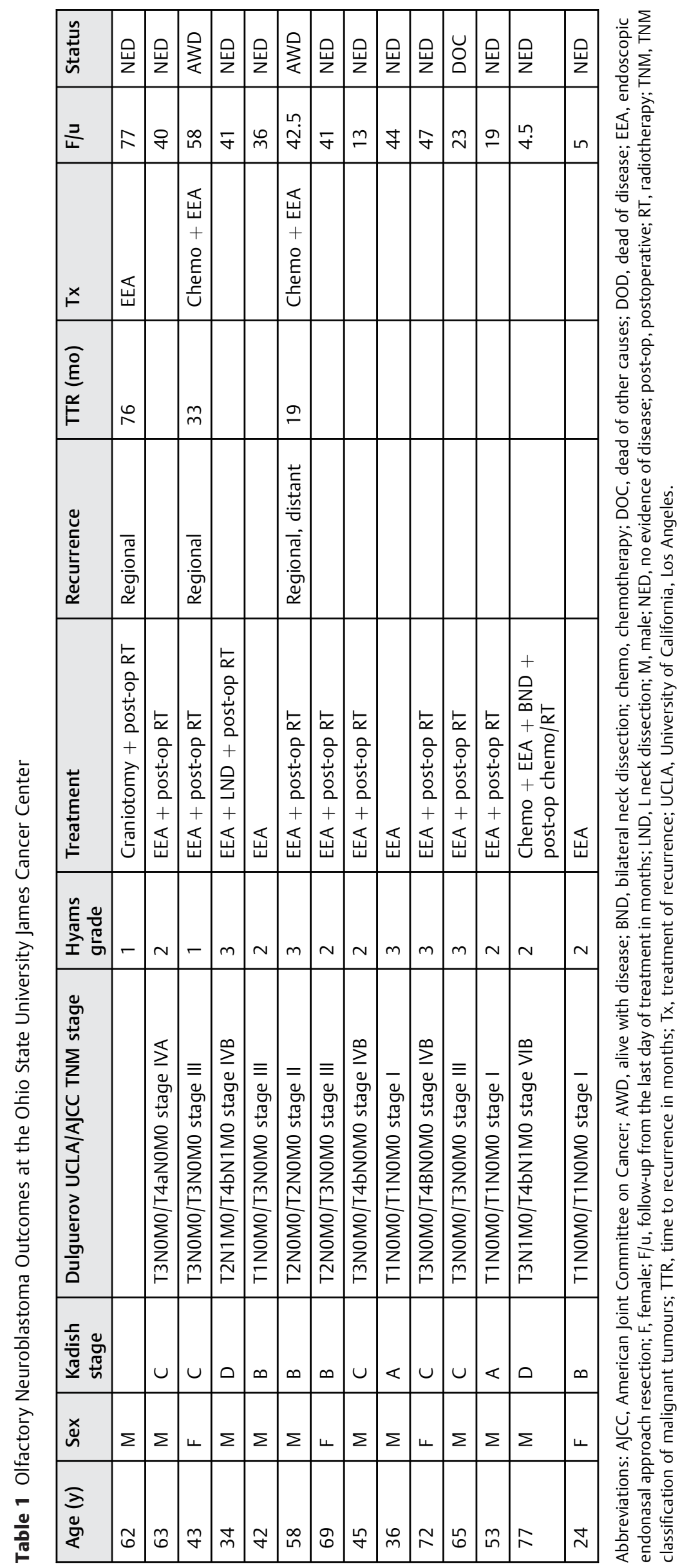




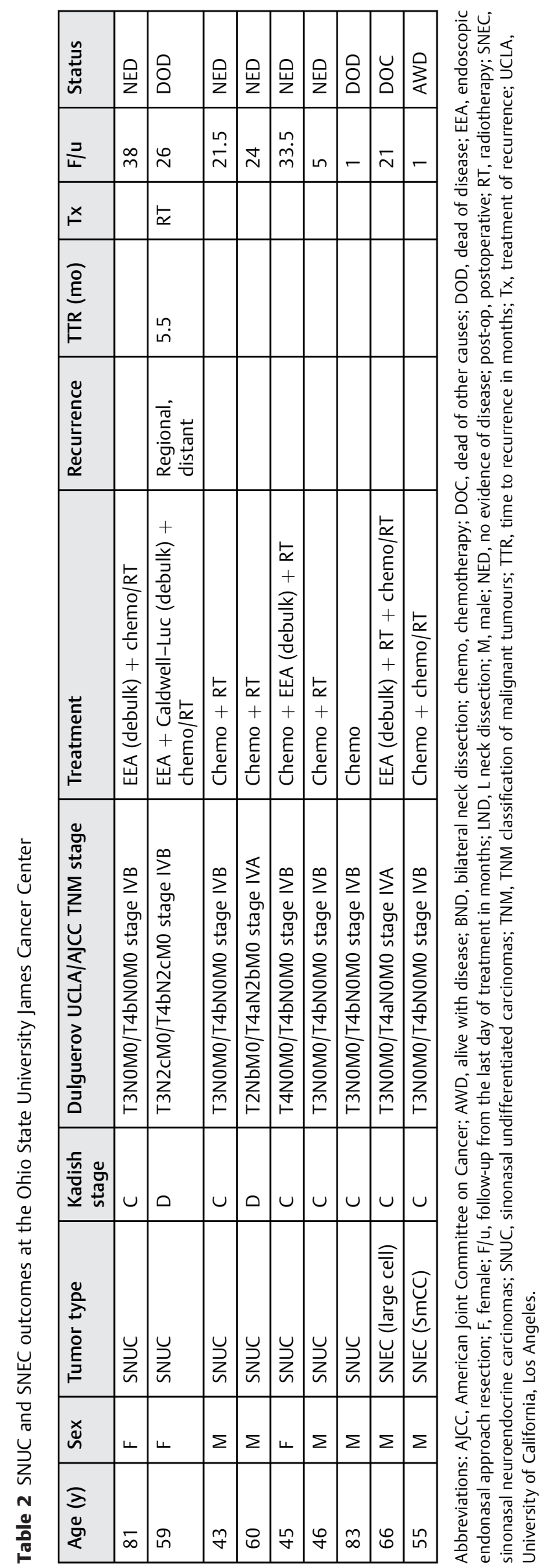


by Hyams. However, these features are absent in high-grade ONBs. Fibrillary cytoplasmic background is a diagnostically helpful microscopic feature seen in $85 \%$ of ONB cases. ${ }^{8}$

A distinction between poorly differentiated variants of ONBs, SNECs, or SNUCs can be particularly difficult. Some authors consider Hyams grade III/IV ONBs and SNUC to be the same entity, although this notion is controversial as the first grading system for ONB, the Hyams grading system, preceded the first mention of SNUC by 10 years. ${ }^{10}$

SNUCs are comprised of pleomorphic cells organized into sheets, large nests, trabeculae, and ribbons with frequent necrosis and no differentiation on light microscopy. Highmitotic activity, atypia, and lymph-vascular invasion can be observed. ${ }^{7,11,12}$

Immunohistochemical markers aid in the exclusion of other diagnoses, but are rarely diagnostic for SNUCs. Strong positivity for cytokeratin with negative synaptophysin and chromogranin staining suggests the diagnosis of SNUC. Although SNUCs often lack neuroendocrine features, neuronspecific enolase-positive staining can be seen. Neuron-specific enolase is a nonspecific marker and is not considered to be diagnostic for true neuroendocrine neoplasms. However, when expressed in SNUC tumors, it can differentiate them from ONBs based on the pattern of staining. Unlike ONB, which stains diffusely positive for neuron-specific enolase, SNUC usually exhibits focal neuron-specific enolase positivity. ${ }^{7,13,14}$

A recent study by Stelow et al reported that some SNUCs could be categorized as a nuclear protein in testis (NUT) midline carcinomas. ${ }^{15}$ These tumors are undifferentiated but may have focal squamous differentiation, which has been previously noted in SNUC tumors and was later suggested as a potential addition to the histological diagnostic criteria for SNUC. ${ }^{12,15}$ Nonetheless, SNUCs and NUT midline carcinomas are classified as two separate malignancies, and clinically most SNUCs are highly responsive to chemotherapy, whereas NUT midline carcinomas are not.

SNEC is often used as an umbrella term, which includes both typical and atypical carcinoid tumors of sinonasal space as well as poorly differentiated SmCC and large cell neuroendocrine carcinomas. The latter malignancy is extremely rare and can be distinguished from moderately differentiated atypical carcinoid tumors by the presence of high-mitotic activity. ${ }^{3} \mathrm{SmCC}$ is morphologically identical to the small cell carcinoma of the lung. In some cases SmCCs have a characteristic appearance on light microscopy in addition to the distinct immunohistochemical profile, which can help distinguish them from other tumors with neuroendocrine features. ${ }^{14}$ In the past, some authors argued that SmCC should be classified as SNUC ${ }^{7}$; however, it is widely accepted that SNUCs and SNECs are two separate entities. ${ }^{8,14}$

In contrast to well-differentiated ONBs, SNEC tumors lack rosette formation. With the exception of SmCC, SNEC tumor cells are larger than ONB cells, have bigger nucleoli, abundant cytoplasm, and neurosecretory granules, while lacking neurofibrillary background. ${ }^{16}$ These distinguishing features are not as helpful when the differential diagnosis includes poorly differentiated ONB as they may lack rosettes or neurofibrillary background. In contrast to SNUCs, SNECs are often positive for chromogranin and synaptophysin. However, there are cases in which a distinction between SNEC and SNUC cannot be made. ${ }^{7,12,14}$

Poorly differentiated ONBs are difficult to distinguish from other sinonasal malignancies. The differential diagnosis is broad and includes malignant melanoma, rhabdomyosarcoma, Ewing sarcoma (ES, primitive neuroectodermal tumor or PNET), malignant lymphoma, and extramedullary plasmacytoma among others. Tumor cell positivity for neuronal markers (chromogranin, synaptophysin), S-100 in sustentacular cells, diffuse positivity for neuron-specific enolase, with absence of epithelial, mesenchymal, myogenic and leukocytic markers is characteristic of ONB ( - Table 3 ). However, if there is a coexpression of neuroendocrine markers and cytokeratin, typical for simple epithelia, neuroendocrine carcinoma is more likely.

Sinonasal malignant melanomas are often amelanotic and can be composed of small blue cells resembling ONBs. Immunohistochemical markers for melanoma, such as

Table 3 Immunohistochemical markers in sinonasal malignancies

\begin{tabular}{|l|l|l|l|l|l|l|l|l|l|l|l|}
\hline Malignancy & SYN & CHR & NSE & CK & S-100 & HMB45 & DES & MSA & VIM & CD99 & LCA \\
\hline ONB & + & + & $+^{\mathrm{a}}$ & - & $+^{\mathrm{b}}$ & - & - & & & - & - \\
\hline SNUC & $-^{*}$ & $-{ }^{*}$ & $+^{\mathrm{a}}$ & + & - & - & - & - & - & $\mathrm{V}$ & - \\
\hline SNEC & $+^{*}$ & $+^{*}$ & + & + & $-^{*}$ & - & - & - & - & $\mathrm{V}$ & - \\
\hline Melanoma & - & - & - & - & $+^{\mathrm{b}}$ & + & - & - & $\mathrm{V}$ & - & - \\
\hline Rhabdomyosarcoma & - & - & - & $\mathrm{V}$ & $\mathrm{V}$ & - & + & + & $+^{*}$ & $\mathrm{~V}$ & \\
\hline ES/PNET & $+^{*}$ & $+^{*}$ & $-{ }^{*}$ & $\mathrm{~V}$ & $\mathrm{~V}$ & - & $\mathrm{V}$ & $\mathrm{V}$ & $+^{*}$ & + & - \\
\hline Lymphoma & - & - & - & - & - & - & - & & & + & + \\
\hline
\end{tabular}

Abbreviations: CD99, product of MIC-2 gene; CHR, chromogranin; CK, cytokeratin; DES, desmin; HMB45, melanoma-associated marker; LCA, leukocyte antigen; MSA, muscle-specific actin; NSE, neuron-specific enolase; S-100, S-100 protein; SYN, synaptophysin; VIM, vimentin.

Note: Based in part on data from Haas and Ganzer. ${ }^{63}$

+ , positive; - , negative; $\mathrm{V}$, variable; $+^{*}$, positive in most cases; $-^{*}$, negative in most cases.

${ }^{a}$ NSE staining is diffuse in ONBs and focal in SNUCs.

bS-100 is positive in sustentacular cells only in ONBs and stains diffusely in melanoma. 
Sox100, S-100, HMB-45, and melanin A are helpful in diagnosis of these tumors. ${ }^{17}$ Sox100 is a highly sensitive and specific immunohistochemical marker for melanoma. It is nearly always expressed in sinonasal melanomas, and is often positive when other markers characteristic of melanoma are negative. ${ }^{18,19}$ In low-grade ONBs with the classic rosette architecture and the presence of sustentacular cells, the location of S-100 positive cells can distinguish these tumors from sinonasal malignant melanoma. The $\mathrm{S}-100$ positive cells in ONBs are limited to the sustentacular cells at the periphery of the cell nests, and melanomas are diffusely positive for S100 protein. ${ }^{3}$ Additionally, tumor location might be helpful in these situations with malignant melanomas limited to the lower part of the nasal cavity beyond the distribution of the olfactory epithelium that gives rise to ONBs. ${ }^{3}$

ES/PNET is another mimic of ONB, with the similar microscopic appearance of small, undifferentiated cell sheets, Homer-Wright rosettes and centrally located fibrils. However, unlike ES/PNET tumors that are CD99 positive in more than $95 \%$ cases and FLI 1 immunopositive in $71 \%$ of cases, virtually all ONB tumors are CD99 and FLI1 negative. ${ }^{15}$ Approximately $85 \%$ of the ES/PNET tumors show characteristic (t [11; 22] [q24; q12]) translocation with EWS and FLI1 gene fusion, which can be confirmed by fluorescence in situ hybridization. $^{16}$

\section{Treatment and Outcomes}

\section{Olfactory Neuroblastoma}

Rosenthal et al first described the classification of sinonasal malignancies with neuroendocrine features into ONB and non-ONB subtypes reporting significant differences in outcomes between the two categories. ${ }^{6}$ Although the treatment of SNUC and carcinoid type SNEC tumors is similar, the management approach to ONBs and poorly differentiated SmCC and large cell type SNECs is vastly different. ${ }^{19}$ Therefore, a thorough assessment and accurate diagnosis are important for the treatment selection and prognosis.

ONBs have a better prognosis and survival compared with other sinonasal neuroendocrine malignancies. In general, a combination of surgery (craniofacial resection) and radiotherapy has shown superior results to radiation alone and has become a standard of care for all resectable cases of ONBs. ${ }^{20-26}$

Traditionally, ONB resections involved an open craniofacial approach. Dulguerov et al reported a meta-analysis of 390 patients treated with an anterior craniofacial approach yielding a 5 -year disease-free survival of $45 \%$ for surgery and radiation. ${ }^{20}$ Conversely, the median overall survival for a case series of 70 patients treated at MD Anderson was 126.3 months (10.5 years) with a median disease-specific survival of 139 months (11.6 years). ${ }^{27}$

Recent advances in endoscopic techniques have led to an increased use of endoscopic resection for ONBs, in an effort to avoid brain manipulation and external facial incisions. Multiple case series from around the world have yielded promising results with similar outcomes to that of open approach. ${ }^{28-33}$ Several small case series reported 3- and 5-year survival range of 89 to $100 \% .{ }^{34-39}$ A meta-analysis of 23 publications comparing endoscopic surgery to open approach showed a $25 \%$ improvement in 10-year overall survival with endoscopic approach. However, these results have to be interpreted with caution due to a strong selection bias; a larger number of patients with advanced disease (Kadish C-D) were treated by open craniofacial approach than by endoscopic approach.

In this study, all ONB cases, including Kadish C and D tumors, were treated with endoscopic resection. With the exception of two T1 Kadish B tumors, all ONB patients underwent postoperative radiation therapy. A neck dissection was only performed for clinically positive nodes. One patient, who was initially treated with an open approach at another institution, underwent an endoscopic resection for regional tumor recurrence at our institution. Two patients with regional and regional-distant recurrence underwent endoscopic resection combined with chemotherapy.

The 2-year disease-free survival for 11 ONB patients with a minimum of 2 -year follow-up was $81 \%$ (9/11). One patient with Dulguerov/UCLA T2N0M0 stage and AJCC T2N0M0 stage II ONB was found to have regional and distant metastases 19 months after the initial treatment. This patient was still alive 23 months after the treatment of the recurrence and 42 months after the initial treatment. One patient with Dulguerov/UCLA T3NOM0 stage and AJCC T3NOM0 stage III ONB died of brain radionecrosis following postoperative proton radiation therapy. Despite these results and the seemingly superior outcomes reported in the literature following endoscopic resection, long-term follow-up is needed to further assess the outcomes of endoscopic approach. ONB is known for its propensity to recur later and requires long-term surveillance with previously reported disease-free survival reduction of $34 \%$ on 15-year follow-up. ${ }^{40}$

Chemotherapy as primary treatment for ONBs has shown inferior outcomes. It is primarily reserved for the treatment of nonresectable disease, as neoadjuvant therapy to decrease the size of the tumor and the extent of the brain or orbit manipulation, and as a radiosensitizer. A series of 50 patients treated with neoadjuvant chemoradiation at the University of Virginia showed outcomes comparable to traditional open resection and postoperative radiotherapy. In this series, patients with Kadish A and B ONBs were treated with preoperative radiation, while Kadish $C$ cases received sequential preoperative chemotherapy and radiation 4 to 6 weeks before open approach resection. The 5- and 15-year diseasefree survival rates were 86.5 and $82.6 \%$, respectively. The local-regional recurrence was $24 \%$ and distant recurrence was $10 \%{ }^{14,19,41}$

In our case series, one patient with Kadish C, Dulguerov/ UCLA T3N1M0 disease received neoadjuvant chemotherapy and postoperative chemoradiation following endoscopic resection with bilateral neck dissection; however, follow-up was limited to 6 months.

Some authors suggested combining an elective neck dissection with radiation therapy. ${ }^{24,42,43}$ Most surgeons agree that a neck dissection is only indicated in patients with cervical metastases, arguing that prophylactic treatment 
adds considerable morbidity and should be reserved for salvage therapy. ${ }^{44-47}$ Noh et al reported a reduction in the rate of relapse in patients undergoing elective neck irradiation, especially radiation to level $1 .^{48}$

\section{Sinonasal Undifferentiated Carcinoma}

SNUCs are known for their aggressive nature and often present with locally advanced disease, orbital and periorbital invasion, and intracranial extension. Surgical management of SNUCs is challenging and the indications for resection vary from institution to institution.

Despite poor outcomes reported in early studies, ${ }^{13}$ recent reports have shown 5 -year overall survival rates between 40 and $75 \% .{ }^{49-52}$ Due to the rarity of these tumors, case series lack uniform treatment paradigms, which complicates the comparison of outcome. Multimodality treatment with or without craniofacial resection (in cases of resectable disease) has yielded improved outcomes. ${ }^{8,19,49,51-53}$ Most series reported utilizing radiotherapy or chemotherapy and radiation in addition to surgery, but no paradigm has demonstrated clear therapeutic advantage.

Currently, the trend is to treat patients presenting with advanced SNUC with combined chemotherapy and radiation leaving surgery as a salvage option. Tanzler et al reported on 15 patients, 9 of whom underwent open resection with subsequent radiation or chemotherapy with 3-year overall survival of $67 \%{ }^{51}$ In a case series of 16 patients half of whom received neoadjuvant chemotherapy and the other half received surgery, the overall survival was $62.5 \%{ }^{8}$ Similarly, a 2year overall survival rate of $64 \%$ was reported by the University of Virginia in a series of 10 patients treated with neoadjuvant chemotherapy followed by radiotherapy. ${ }^{49,51,54}$

Conversely, Al-Mamgani at al showed superior outcomes with a 5 -year overall survival rate of $74 \%$ when a tailored approach was utilized with surgery and postoperative radiation or chemoradiation used for resectable disease and neoadjuvant chemotherapy or chemoradiation alone used for nonresectable cases. ${ }^{48}$ Multimodality treatment for SNUC has been proven to improve survival regardless of the primary treatment. $^{50,55}$

More recently, small case series treated with endoscopic resection reported comparable results to the open approach with local and regional control of up to $78 \%$ for endoscopic resection followed by radiation. ${ }^{56}$ Surgery as primary treatment in combination with neoadjuvant therapy showed improved survival and local-regional control. ${ }^{55}$

In this study, three SNUC cases underwent endoscopic gross total removal followed by chemotherapy and radiation therapy (two patients had an initial diagnosis of ONB). All of our cases were treated with chemotherapy and radiation. The 2 -year disease-free survival for the four patients was $75 \%$.

\section{Sinonasal Neuroendocrine Carcinomas}

Typical and atypical carcinoids are generally considered separately from SmCC and large cell neuroendocrine carcinoma as their treatment and outcomes differ significantly. A limited number of prior studies with the lack of uniform treatment protocols for these malignancies make outcome comparison difficult. ${ }^{54,57,58}$ Some centers favor treating patients with these malignancies similar to those with SNUCs and others use treatment paradigms similar to those used for patients with ONBs.

A recent SNEC case series showed a 5-year overall survival of $66.9 \%$, although the authors did not distinguish between the histopathological types of SNEC. This study included patients with various treatment strategies that were based on the interdisciplinary consensus with nearly half the patients undergoing surgery for definitive management of their disease $^{59}$ As with SNUCs, neoadjuvant chemotherapy followed by surgery or chemoradiation is a preferred strategy due to high chemosensitivity of most SNEC cases. A complete response to neoadjuvant chemotherapy correlated with improved survival in prior studies. ${ }^{8,59-62}$

SmCC is a rare malignancy with outcomes that are inferior to those of other sinonasal neuroendocrine cancers, but comparable to the outcomes of small cell carcinoma at other sites. It is difficult to recommend a single treatment strategy since prior studies lacked a uniform treatment approach. Chemoresponsive cases showed better 3-year survival. ${ }^{57}$ Neoadjuvant chemotherapy with subsequent chemoradiation or surgery and postoperative radiation have been utilized in previous studies. ${ }^{54,57,58}$

One SNEC patient at our institution had a diagnostically challenging histopathological profile with variable reactivity for cytokeratin, synaptophysin, chromogranin, peripheral sustentacular S-100 protein, and neuron-specific enolase. Although the pattern of S-100 staining was similar to that of ONB and the overall findings did not show classic features of poorly differentiated neuroendocrine carcinoma (SmCC or large cell type), an outside pathological consultation suggested to manage this tumor as a sinonasal SmCC. Therefore, this lesion was staged as T4bNOM0, AJCC stage IVB neuroendocrine carcinoma and was treated with neoadjuvant chemotherapy followed by the chemotherapy and radiation. At the time of this report the follow-up was insufficient.

Our study included only one patient with T4aNOMO, AJCC stage IVA large cell neuroendocrine carcinoma. The patient underwent neoadjuvant chemotherapy and radiation before endoscopic gross total removal with postoperative radiation treatment. Unfortunately, this patient died due to brain radionecrosis secondary to proton radiation therapy.

Large cell neuroendocrine cancer is extremely rare; thus, data regarding this tumor are sparse in the literature. Based on the existing reports, these malignancies have poor outcomes comparable to that of SmCC. Aggressive multimodality therapy is usually applied, but the outcomes remain poor.

\section{Conclusions}

Sinonasal malignancies with neuroendocrine features cannot be distinguished without a thorough histopathological evaluation. Individualized assessment and treatment strategies that take into account tumor stage, grade, and histopathological characteristics can improve the accuracy of the initial assessment and treatment outcomes of sinonasal carcinomas with neuroendocrine features. Multicenter studies and 
collaboration with uniform treatment protocols are essential to improve the reporting of treatment outcomes. Endoscopic resection of these malignancies has shown outcomes similar to previously reported open resections, but long-term followup is needed to further assess the outcomes.

\section{References}

1 Bell D, Hanna EY, Weber RS, et al. Neuroendocrine neoplasms of the sinonasal region. Head Neck 2015 (e-pub ahead of print). doi: 10.1002/hed.24152

2 Perez-Ordonez B. Neuroendocrine tumors. In: Barnes L, Eveson JW, Reichart P, Sidransky D, eds. Pathology and Genetics of Head and Neck Tumours. World Health Organization Classification of Tumours. Vol 9. Lyon, France: IARC Press; 2005:26-27

3 Xu B, Chetty R, Perez-Ordoñez B. Neuroendocrine neoplasms of the head and neck: some suggestions for the new WHO classification of head and neck tumors. Head Neck Pathol 2014;8(1):24-32

4 van der Laan TP, Bij HP, van Hemel BM, et al. The importance of multimodality therapy in the treatment of sinonasal neuroendocrine carcinoma. Eur Arch Otorhinolaryngol 2013;270(9): 2565-2568

5 Mills SE. Neuroectodermal neoplasms of the head and neck with emphasis on neuroendocrine carcinomas. Mod Pathol 2002;15(3): 264-278 Review

6 Rosenthal DI, Barker JL Jr, El-Naggar AK, et al. Sinonasal malignancies with neuroendocrine differentiation: patterns of failure according to histologic phenotype. Cancer 2004;101(11):2567-2573

7 Su SY, Bell D, Hanna EY. Esthesioneuroblastoma, neuroendocrine carcinoma, and sinonasal undifferentiated carcinoma: differentiation in diagnosis and treatment. Int Arch Otorhinolaryngol 2014; 18(Suppl 2):S149-S156

8 Iezzoni JC, Mills SE. "Undifferentiated" small round cell tumors of the sinonasal tract: differential diagnosis update. Am J Clin Pathol 2005;124(Suppl):S110-S121

9 Ow TJ, Bell D, Kupferman ME, Demonte F, Hanna EY. Esthesioneuroblastoma. Neurosurg Clin N Am 2013;24(1):51-65

10 Faragalla H, Weinreb I. Olfactory neuroblastoma: a review and update. Adv Anat Pathol 2009;16(5):322-331

11 Frierson HF Jr, Mills SE, Fechner RE, Taxy JB, Levine PA. Sinonasal undifferentiated carcinoma. An aggressive neoplasm derived from schneiderian epithelium and distinct from olfactory neuroblastoma. Am J Surg Pathol 1986;10(11):771-779

12 Ejaz A, Wenig BM. Sinonasal undifferentiated carcinoma: clinical and pathologic features and a discussion on classification, cellular differentiation, and differential diagnosis. Adv Anat Pathol 2005; 12(3):134-143

13 Cerilli LA, Holst VA, Brandwein MS, Stoler MH, Mills SE. Sinonasal undifferentiated carcinoma: immunohistochemical profile and lack of EBV association. Am J Surg Pathol 2001;25(2):156-163

14 Smith SR, Som P, Fahmy A, Lawson W, Sacks S, Brandwein M. A clinicopathological study of sinonasal neuroendocrine carcinoma and sinonasal undifferentiated carcinoma. Laryngoscope 2000; 110(10 Pt 1):1617-1622

15 Stelow EB, Bellizzi AM, Taneja K, et al. NUT rearrangement in undifferentiated carcinomas of the upper aerodigestive tract. Am J Surg Pathol 2008;32(6):828-834

16 Rischin D, Coleman A. Sinonasal malignancies of neuroendocrine origin. Hematol Oncol Clin North Am 2008;22(6):1297-1316, xi

17 Montone KT. The differential diagnosis of sinonasal/nasopharyngeal neuroendocrine/neuroectodermally derived tumors. Arch Pathol Lab Med 2015;139(12):1498-1507

18 Ordóñez NG. Value of SOX10 immunostaining in tumor diagnosis. Adv Anat Pathol 2013;20(4):275-283

19 Miettinen M, McCue PA, Sarlomo-Rikala M, et al. Sox10-a marker for not only schwannian and melanocytic neoplasms but also myoepithelial cell tumors of soft tissue: a systematic analysis of 5134 tumors. Am J Surg Pathol 2015;39(6):826-835

20 Dulguerov P, Allal AS, Calcaterra TC. Esthesioneuroblastoma: a meta-analysis and review. Lancet Oncol 2001;2(11):683-690

21 Ingeholm P, Theilgaard SA, Buchwald C, Hansen HS, Francis D. Esthesioneuroblastoma: a Danish clinicopathological study of 40 consecutive cases. APMIS 2002;110(9):639-645

22 Jethanamest D, Morris LG, Sikora AG, Kutler DI. Esthesioneuroblastoma: a population-based analysis of survival and prognostic factors. Arch Otolaryngol Head Neck Surg 2007;133(3):276-280

23 Gruber G, Laedrach K, Baumert B, Caversaccio M, Raveh J, Greiner R. Esthesioneuroblastoma: irradiation alone and surgery alone are not enough. Int J Radiat Oncol Biol Phys 2002;54(2):486-491

24 Chao KS, Kaplan C, Simpson JR, et al. Esthesioneuroblastoma: the impact of treatment modality. Head Neck 2001;23(9):749-757

25 Ozsahin M, Gruber G, Olszyk O, et al. Outcome and prognostic factors in olfactory neuroblastoma: a rare cancer network study. Int J Radiat Oncol Biol Phys 2010;78(4):992-997

26 Devaiah AK, Andreoli MT. Treatment of esthesioneuroblastoma: a 16-year meta-analysis of 361 patients. Laryngoscope 2009; 119(7):1412-1416

27 Ow TJ, Hanna EY, Roberts DB, et al. Optimization of long-term outcomes for patients with esthesioneuroblastoma. Head Neck 2014;36(4):524-530

28 Zafereo ME, Fakhri S, Prayson R, et al. Esthesioneuroblastoma: 25year experience at a single institution. Otolaryngol Head Neck Surg 2008;138(4):452-458

29 Dave SP, Bared A, Casiano RR. Surgical outcomes and safety of transnasal endoscopic resection for anterior skull tumors. Otolaryngol Head Neck Surg 2007;136(6):920-927

30 Liu JK, O'Neill B, Orlandi RR, Moscatello AL, Jensen RL, Couldwell WT. Endoscopic-assisted craniofacial resection of esthesioneuroblastoma: minimizing facial incisions-technical note and report of 3 cases. Minim Invasive Neurosurg 2003;46(5):310-315

31 Podboj J, Smid L. Endoscopic surgery with curative intent for malignant tumors of the nose and paranasal sinuses. Eur J Surg Oncol 2007;33(9):1081-1086

32 Poetker DM, Toohill RJ, Loehrl TA, Smith TL. Endoscopic management of sinonasal tumors: a preliminary report. Am J Rhinol 2005; 19(3):307-315

33 Suriano M, De Vincentiis M, Colli A, Benfari G, Mascelli A, Gallo A. Endoscopic treatment of esthesioneuroblastoma: a minimally invasive approach combined with radiation therapy. Otolaryngol Head Neck Surg 2007;136(1):104-107

34 Unger F, Haselsberger K, Walch C, Stammberger H, Papaefthymiou G. Combined endoscopic surgery and radiosurgery as treatment modality for olfactory neuroblastoma (esthesioneuroblastoma). Acta Neurochir (Wien) 2005;147(6):595-601, discussion 601-602

35 Castelnuovo PG, Delù G, Sberze F, et al. Esthesioneuroblastoma: endonasal endoscopic treatment. Skull Base 2006;16(1):25-30

36 Lund V, Howard DJ, Wei WI. Endoscopic resection of malignant tumors of the nose and sinuses. Am J Rhinol 2007;21(1):89-94

37 Nicolai P, Battaglia P, Bignami M, et al. Endoscopic surgery for malignant tumors of the sinonasal tract and adjacent skull base: a 10-year experience. Am J Rhinol 2008;22(3):308-316

38 Folbe A, Herzallah I, Duvvuri U, et al. Endoscopic endonasal resection of esthesioneuroblastoma: a multicenter study. Am J Rhinol Allergy 2009;23(1):91-94

39 De Bonnecaze G, Chaput B, Al Hawat A, et al. Long-term oncological outcome after endoscopic surgery for olfactory esthesioneuroblastoma. Acta Otolaryngol 2014;134(12):1259-1264

40 Howard DJ, Lund VJ, Wei WI. Craniofacial resection for tumors of the nasal cavity and paranasal sinuses: a 25 -year experience. Head Neck 2006;28(10):867-873

41 Polin RS, Sheehan JP, Chenelle AG, et al. The role of preoperative adjuvant treatment in the management of esthesioneuroblastoma: the University of Virginia experience. Neurosurgery 1998; 42(5):1029-1037 
42 Beitler JJ, Fass DE, Brenner HA, et al. Esthesioneuroblastoma: is there a role for elective neck treatment? Head Neck 1991;13(4): 321-326

43 Monroe AT, Hinerman RW, Amdur RJ, Morris CG, Mendenhall WM. Radiation therapy for esthesioneuroblastoma: rationale for elective neck irradiation. Head Neck 2003;25(7):529-534

44 Diaz EM Jr, Johnigan RH III, Pero C, et al. Olfactory neuroblastoma: the 22-year experience at one comprehensive cancer center. Head Neck 2005;27(2):138-149

45 Loy AH, Reibel JF, Read PW, et al. Esthesioneuroblastoma: continued follow-up of a single institution's experience. Arch Otolaryngol Head Neck Surg 2006;132(2):134-138

46 Lund VJ, Howard DJ, Wei WI, Cheesman AD. Craniofacial resection for tumors of the nasal cavity and paranasal sinuses-a 17-year experience. Head Neck 1998;20(2):97-105

47 Zanation AM, Ferlito A, Rinaldo A, et al. When, how and why to treat the neck in patients with esthesioneuroblastoma: a review. Eur Arch Otorhinolaryngol 2010;267(11):1667-1671

48 Noh OK, Lee SW, Yoon SM, et al. Radiotherapy for esthesioneuroblastoma: is elective nodal irradiation warranted in the multimodality treatment approach? Int J Radiat Oncol Biol Phys 2011; 79(2):443-449

49 Musy PY, Reibel JF, Levine PA. Sinonasal undifferentiated carcinoma: the search for a better outcome. Laryngoscope 2002;112 (8 Pt 1):1450-1455

50 Al-Mamgani A, van Rooij P, Mehilal R, Tans L, Levendag PC. Combined-modality treatment improved outcome in sinonasal undifferentiated carcinoma: single-institutional experience of 21 patients and review of the literature. Eur Arch Otorhinolaryngol 2013;270(1):293-299

51 Tanzler ED, Morris CG, Orlando CA, Werning JW, Mendenhall WM. Management of sinonasal undifferentiated carcinoma. Head Neck 2008;30(5):595-599

52 Chen AM, Daly ME, El-Sayed I, et al. Patterns of failure after combined-modality approaches incorporating radiotherapy for sinonasal undifferentiated carcinoma of the head and neck. Int J Radiat Oncol Biol Phys 2008;70(2):338-343
53 Miyamoto RC, Gleich LL, Biddinger PW, Gluckman JL. Esthesioneuroblastoma and sinonasal undifferentiated carcinoma: impact of histological grading and clinical staging on survival and prognosis. Laryngoscope 2000;110(8):1262-1265

54 Perez-Ordonez B, Caruana SM, Huvos AG, Shah JP. Small cell neuroendocrine carcinoma of the nasal cavity and paranasal sinuses. Hum Pathol 1998;29(8):826-832

55 Reiersen DA, Pahilan ME, Devaiah AK. Meta-analysis of treatment outcomes for sinonasal undifferentiated carcinoma. Otolaryngol Head Neck Surg 2012;147(1):7-14

56 Revenaugh PC, Seth R, Pavlovich JB, Knott PD, Batra PS. Minimally invasive endoscopic resection of sinonasal undifferentiated carcinoma. Am J Otolaryngol 2011;32(6):464-469

57 Fitzek MM, Thornton AF, Varvares M, et al. Neuroendocrine tumors of the sinonasal tract. Results of a prospective study incorporating chemotherapy, surgery, and combined proton-photon radiotherapy. Cancer 2002;94(10):2623-2634

58 Babin E, Rouleau V, Vedrine PO, et al. Small cell neuroendocrine carcinoma of the nasal cavity and paranasal sinuses. J Laryngol Otol 2006;120(4):289-297

59 Mitchell EH, Diaz A, Yilmaz T, et al. Multimodality treatment for sinonasal neuroendocrine carcinoma. Head Neck 2012;34(10): 1372-1376

60 Deutsch BD, Levine PA, Stewart FM, Frierson HF Jr, Cantrell RW. Sinonasal undifferentiated carcinoma: a ray of hope. Otolaryngol Head Neck Surg 1993;108(6):697-700

61 Gallo O, Graziani P, Fini-Storchi O. Undifferentiated carcinoma of the nose and paranasal sinuses. An immunohistochemical and clinical study. Ear Nose Throat J 1993;72(9):588-590, 593-595

62 Levine PA, Frierson HF Jr, Stewart FM, Mills SE, Fechner RE, Cantrell RW. Sinonasal undifferentiated carcinoma: a distinctive and highly aggressive neoplasm. Laryngoscope 1987;97(8 Pt 1):905-908

63 Haas I, Ganzer U. Does sophisticated diagnostic workup on neuroectodermal tumors have an impact on the treatment of esthesioneuroblastoma? Onkologie 2003;26(3):261-267 